\title{
(6) \\ Screen time is associated with adiposity and insulin resistance in children
} OPEN ACCESS

\author{
Claire M Nightingale, ${ }^{1}$ Alicja R Rudnicka, ${ }^{1}$ Angela S Donin, ${ }^{1}$ Naveed Sattar, ${ }^{2}$ \\ Derek G Cook, ${ }^{1}$ Peter H Whincup, ${ }^{1}$ Christopher G Owen ${ }^{1}$
}

\begin{abstract}
- Additional material is published online only. To view please visit the journal online (http://dx.doi.org/10.1136/ archdischild-2016-312016).

${ }^{1}$ Population Health Research Institute, St George's, University of London, London, UK ${ }^{2}$ Institute of Cardiovascular \& Medical Sciences, University of Glasgow, Glasgow, UK
\end{abstract}

Correspondence to Dr Claire M Nightingale, Population Health Research Institute, St. George's, University of London, London SW17 ORE, UK; cnightin@sgul.ac.uk

Received 15 September 2016 Revised 28 November 2016 Accepted 4 January 2017 Published Online First 6 February 2017
ABSTRACT
Background Higher screen time is associated with

type 2 diabetes (T2D) risk in adults, but the association with $\mathrm{T} 2 \mathrm{D}$ risk markers in children is unclear. We examined associations between self-reported screen time and $\mathrm{T} 2 \mathrm{D}$ risk markers in children.

Methods Survey of 4495 children aged 9-10 years who had fasting cardiometabolic risk marker assessments, anthropometry measurements and reported daily screen time; objective physical activity was measured in a subset of 2031 children.

Results Compared with an hour or less screen time daily, those reporting screen time over 3 hours had higher ponderal index $(1.9 \%, 95 \% \mathrm{Cl} 0.5 \%$ to $3.4 \%)$, skinfold thickness $(4.5 \%, 0.2 \%$ to $8.8 \%)$, fat mass index $(3.3 \%, 0.0 \%$ to $6.7 \%)$, leptin $(9.2 \%, 1.1 \%$ to $18.0 \%)$ and insulin resistance $(10.5 \%, 4.9 \%$ to $16.4 \%)$; associations with glucose, $\mathrm{HbA}_{1 c}$, physical activity and cardiovascular risk markers were weak or absent. Associations with insulin resistance remained after adjustment for adiposity, socioeconomic markers and physical activity.

Conclusions Strong graded associations between screen time, adiposity and insulin resistance suggest that reducing screen time could facilitate early $T 2 D$ prevention. While these observations are of considerable public health interest, evidence from randomised controlled trials is needed to suggest causality.

\section{INTRODUCTION}

The global prevalence of type 2 diabetes (T2D), overweight and obesity has been increasing in adults as well as adolescents and children. ${ }^{1}{ }^{2}$ Awareness of the early determinants of adiposity and T2D risk in young people may be important for reducing risks of T2D and obesity across the life course. The health effects of activities that encourage sedentary behaviour, such as time spent watching television, using computers or games consoles (together referred to as 'screen time') have been a particular focus. Increased screen time has been prospectively associated with adiposity ${ }^{3}$ and T2D risk in adults. ${ }^{4}$ Studies have shown graded positive associations between prolonged screen time and adiposity in childhood. ${ }^{5}{ }^{6}$ However, less is known about the effects of prolonged screen time on T2D risk markers in childhood, particularly insulin resistance (IR) and glycaemia. Such associations could be of public health importance in providing evidence-based recommendations for healthy screen-time duration, especially as recent trends suggest that screen time is increasing in childhood. ${ }^{7}$ We therefore examined the association

\section{What is already known on this topic?}

Increased screen time is prospectively associated with adiposity and type 2 diabetes in adults.

- Evidence suggests graded associations between screen time and adiposity in children.

\section{What this study adds?}

- This study demonstrated strong graded positive associations between screen time, adiposity and risk markers for type 2 diabetes (particularly insulin resistance) in children.

- The associations between screen time and insulin resistance markers were largely independent of socioeconomic status, pubertal status, objectively measured physical activity and adiposity.

between self-reported daily screen time and risk markers for T2D and cardiovascular disease, in a large-scale multi-ethnic population-based study of children aged $9-10$ years.

\section{METHODS}

The Child Heart and Health Study in England (CHASE) was a cross-sectional survey of cardiovascular health in UK schoolchildren of white European, black African-Caribbean and South Asian origin aged 9-10 years carried out in a sample of 200 primary schools in London, Birmingham and Leicester; full details of the study have been reported elsewhere. ${ }^{8-10}$ Ethical approval for the study was obtained from the relevant multicentre ethics committee. A single survey team carried out all measurements between October 2004 and February 2007. Children's ethnic origin was based on the ethnicity of both parents or (where not available) the parentally defined ethnicity of the child; in a small proportion of children where this was not available (1\%), child information on the place of birth of parents and grandparents was used to define ethnicity. Ethnic group was classified as 'white European', which includes those of white European including white British ethnicity; 'black African-Caribbean', which includes black African, black Caribbean and black other; 'South Asian', which includes Indian, Pakistani, 
Bangladeshi and South Asian other; those of mixed or other ethnic origins were included in an 'other ethnic groups' category. Socioeconomic status was based on self-reported parental occupation (highest of mother or father) and coded using the National Statistics Socio-economic Classification (NS-SEC). ${ }^{11}$

Height was measured to the last complete millimetre using a portable stadiometer (Chasmors, London, UK) and weight was measured using an electronic digital scale (Tanita, Tokyo, Japan). Ponderal index was calculated as a weight divided by height cubed $\left(\mathrm{kg} / \mathrm{m}^{3}\right)$, providing a weight for height measure which was largely independent of height in the study population. Right-sided skinfolds were measured at four locations (biceps, triceps, subscapular, suprailiac) and summed. Hand-to-foot bioimpedance was measured using the Bodystat 1500 bioimpedance monitor (Bodystat, Isle of Man, UK); fat mass was derived using ethnic and sex-specific validated equations ${ }^{12}$ and presented as a fat mass index (fat mass/height ${ }^{5}$ ), which was independent of height. Blood samples were collected after an overnight fast. $\mathrm{HbA}_{1 \mathrm{c}}$ was analysed in whole blood by ion exchange high-performance liquid chromatography. Plasma glucose was measured using the hexokinase method; triglyceride, high-density lipoprotein (HDL) cholesterol and total cholesterol were measured in serum using an Olympus autoanalyser. Insulin was measured in serum using an ELISA method, ${ }^{13} \mathrm{C}$ reactive protein (CRP) using ultra-sensitive nephelometry and leptin using a radioimmunoassay. Homeostasis model assessment (HOMA) equations provided an estimate of IR. ${ }^{14}$ Seated blood pressure was measured twice in the right arm after 5 min rest using an Omron 907 blood pressure monitor and adjusted for appropriate cuff size ${ }^{15}$; average systolic and diastolic blood pressures were calculated from the two readings. In a subset of children in the last 80 schools studied, objective physical activity counts and time spent in moderate-to-vigorous physical activity (MVPA) were measured using a waist-worn accelerometer
(ActiGraph GT1M, ActiGraph, Pensacola, Florida, USA). ${ }^{9}$ Pubertal status was measured in girls only, using the Tanner breast development score. ${ }^{16}$ Child questionnaires administered on the same day that physical measurements were taken asked, 'How many hours each day do you spend watching television or video and playing computer games?' and children were asked to tick one of the following options: 'none; an hour or less; one to two hours; two to three hours or more than three hours'.

Outcomes following approximately log-normal distributions were log-transformed; geometric means and percentage differences are presented for log-transformed variables. We compared differences in outcomes by self-reported screen time category using those that reported 'an hour or less of screen-time' as a reference group and tested for a linear trend using multilevel linear regression models adjusted for sex, age (in quartiles) ethnicity and month as fixed effects. The intraclass correlation (ICC) for school-level clustering was between 0.02 and 0.09 for all cardiometabolic risk markers and physical activity counts and was higher for time spent in MVPA (ICC $=0.32$ ); hence, it was important to adjust for school as a random effect to account for clustering of children within schools. The effect of adjustment for socioeconomic status (NS-SEC), pubertal status (girls only) and physical activity was assessed because these are potential confounders of the association between screen time and diabetic risk markers. Further adjustment for adiposity was also made, acknowledging that this may be a potential mediator. We examined whether associations between screen time and outcome variables were consistent in boys and girls and in different ethnic groups by testing for an interaction between screen time and sex or ethnic group.

\section{RESULTS}

Of the 8641 children who were invited, 5887 (68\%) took part in the study. Of these, $4884(83 \%)$ provided a fasting blood

Table 1 Adiposity and cardiometabolic risk markers: overall and by sex

\begin{tabular}{|c|c|c|c|}
\hline & Boys $(n=2158)$ & Girls $(n=2337)$ & All participants $(n=4495)$ \\
\hline \multicolumn{4}{|l|}{ Geometric mean $(95 \% \mathrm{Cl})$} \\
\hline Ponderal index $\left(\mathrm{kg} / \mathrm{m}^{3}\right)$ & $13.0(13.0$ to13.1) & $13.2(13.1$ to 13.3$)$ & 13.1 (13.1 to 13.2$)$ \\
\hline Sum of skinfolds (mm) & 36.7 (35.9 to 37.6$)$ & 45.7 (44.7 to 46.7$)$ & 41.1 (40.4 to 41.9$)$ \\
\hline Fat mass index $\left(\mathrm{kg} / \mathrm{m}^{5}\right)$ & 1.91 (1.87 to 1.94$)$ & 2.17 (2.13 to 2.21$)$ & 2.04 (2.01 to 2.07 ) \\
\hline $\mathrm{HbA}_{1 \mathrm{c}}(\%)$ & $5.24(5.22$ to 5.26$)$ & $5.25(5.23$ to 5.26$)$ & 5.24 (5.23 to 5.26$)$ \\
\hline Glucose (mmol/L) & 4.56 (4.54 to 4.58$)$ & 4.47 (4.45 to 4.49 ) & 4.51 (4.50 to 4.53$)$ \\
\hline Insulin (mU/L) & $6.46(6.23$ to 6.69$)$ & 8.14 (7.86 to 8.43$)$ & 7.28 (7.07 to 7.51$)$ \\
\hline HOMA-IR & 0.82 (0.79 to 0.85$)$ & 1.02 (0.99 to 1.06$)$ & 0.92 (0.89 to 0.95$)$ \\
\hline Leptin $(\mathrm{ng} / \mathrm{mL})$ & 7.15 (6.83 to 7.48$)$ & $11.49(11.00$ to 12.00$)$ & 9.15 (8.83 to 9.48$)$ \\
\hline $\mathrm{C}$ reactive protein (mg/L) & $0.44(0.42$ to 0.47$)$ & $0.57(0.54$ to 0.60$)$ & $0.50(0.48$ to 0.53$)$ \\
\hline Triglycerides (mmol/L) & $0.76(0.74$ to 0.77$)$ & 0.85 (0.84 to 0.87$)$ & 0.81 (0.79 to 0.82 ) \\
\hline HDL cholesterol (mmol/L) & 1.53 (1.51 to 1.54$)$ & 1.44 (1.42 to 1.45$)$ & 1.48 (1.47 to 1.49$)$ \\
\hline LDL cholesterol (mmol/L) & 2.60 (2.57 to 2.64$)$ & 2.60 (2.57 to 2.63$)$ & 2.60 (2.58 to 2.63$)$ \\
\hline Total cholesterol (mmol/L) & 4.53 (4.49 to 4.56$)$ & $4.47(4.44$ to 4.50$)$ & 4.50 (4.47 to 4.52 ) \\
\hline \multicolumn{4}{|l|}{ Mean $(95 \% \mathrm{Cl})$} \\
\hline Systolic BP (mm Hg) & $105.2(104.6$ to 105.7$)$ & $104.2(103.7$ to 104.8$)$ & $104.7(104.2$ to 105.2$)$ \\
\hline Diastolic BP $(\mathrm{mm} \mathrm{Hg})$ & $62.8(62.3$ to 63.3$)$ & $63.0(62.5$ to 63.5$)$ & $62.9(62.4$ to 63.3$)$ \\
\hline Daily counts* & 437416 (429 717 to 445114$)$ & 362857 (355 382 to 370332 ) & 397973 (391 604 to 404342 ) \\
\hline Daily time spent in MVPA (mins)* & 78.4 (75.9 to 80.9$)$ & $61.6(59.1$ to 64.1$)$ & 69.5 (67.1 to 71.9$)$ \\
\hline
\end{tabular}


sample. Analyses focused on 4495 children (2337 girls and 2158 boys), average age 9.9 years (95\% reference range 9.2 to 10.7 years), who answered the question on daily screen time, had full anthropometric measurements and a fasting blood sample. Of these, 2031 children also had measurements of physical activity using accelerometery. Participant characteristics are shown in online supplementary table $S 1$, including mean age of participants, gender and ethnic balance, head of household socioeconomic classification and month of measurement. Overall $4 \%$ of the study population reported no screen time, $37 \%$ reported an hour or less, 28\% reported 1-2 hours, 13\% reported 2-3 hours and 18\% reported more than 3 hours of screen time. A larger proportion of boys (22\%) than girls (14\%) reported more than 3 hours of daily screen time. A higher proportion of black African-Caribbeans (23\%) reported more than 3 hours of daily screen time compared with white Europeans (16\%) and South Asians (16\%). Adiposity and cardiometabolic risk markers are shown by sex and overall in table 1 . In general, girls had higher levels of adiposity markers, leptin, fasting insulin, IR, triglyceride, CRP and lower levels of fasting glucose and HDL cholesterol than boys; girls had lower levels of physical activity counts and time spent in MVPA than boys. There were trends between screen time and ponderal index, sum of skinfolds and fat mass index (table 2). Levels of these adiposity variables were higher among children who reported more than 3 hours of screen time compared with those who reported an hour or less of screen time. There was a strong trend between screen time, leptin, fasting insulin and HOMA-IR (table 2). Children who reported more than 3 hours of screen time had higher levels of leptin $(9.2 \%, 95 \%$ CI $1.1 \%$ to $18.0 \%)$, insulin $(10.7 \%, 5.1 \%$ to $16.7 \%)$ and HOMA-IR $(10.5 \%, 4.9 \%$ to
16.4\%) compared with those who reported an hour or less. Adjustment for fat mass index reduced effect sizes for insulin and IR by approximately one-quarter $(7.9 \%, 95 \%$ CI $2.9 \%$ to $13.1 \% ; 7.7 \%, 2.8 \%$ to $13.0 \%$ respectively). There was a borderline significant trend between screen time and triglycerides and physical activity counts and time spent in MVPA. There was no formal evidence of a trend between screen time and $\mathrm{HbA}_{1 \mathrm{c}}$, fasting glucose and other cardiovascular risk factors, including lipids and blood pressure (even with further adjustment for height). Associations between screen time and fasting insulin, IR, ponderal index, sum of skinfolds and fat mass index were not appreciably affected by adjustment for socioeconomic status or physical activity measures in a subset (ie, the magnitude of associations were similar and statistically significant associations remained significant). Adjustment for pubertal status in girls slightly weakened associations for IR markers though associations for fasting insulin and HOMA-IR remained statistically significant; associations for adiposity markers were attenuated though the association with ponderal index remained statistically significant. There was no evidence that associations differed in boys and girls and between ethnic groups (all tests for interaction $\mathrm{p} \geq 0.05$, data available from authors).

\section{DISCUSSION}

The present study showed an association between screen time and measures of adiposity, which has previously been observed in prospective studies of children. ${ }^{5}$ We extend these observations by demonstrating strong graded associations with T2D risk factors, particularly IR (although not glucose or $\mathrm{HbA}_{1 \mathrm{c}}$ ). The association for IR was independent of socioeconomic markers, pubertal status and objectively measured physical

Table 2 Associations between self-reported screen time (television, video and computer games) and adiposity and cardiometabolic risk markers

\begin{tabular}{|c|c|c|c|c|c|c|}
\hline & $\begin{array}{l}\text { None } \\
(n=180)\end{array}$ & $\begin{array}{l}\text { An hour } \\
\text { or less } \\
(n=1664)\end{array}$ & $\begin{array}{l}1-2 \text { hours } \\
(n=1268)\end{array}$ & $\begin{array}{l}2-3 \text { hours } \\
(n=575)\end{array}$ & $\begin{array}{l}\text { More than three hours } \\
(n=808)\end{array}$ & $\begin{array}{l}p \text { (linear } \\
\text { trend) }\end{array}$ \\
\hline \multicolumn{7}{|c|}{ \% difference compared with 'An hour or less self-reported screen time' as the reference group (95\% Cl), p (difference) } \\
\hline Ponderal index $\left(\mathrm{kg} / \mathrm{m}^{3}\right)$ & $-1.2(-3.7$ to 1.3$)$ & 0 & $1.5(0.3$ to 2.8$)$ & $1.0(-0.6$ to 2.6$)$ & $1.9(0.5$ to 3.4$)$ & 0.003 \\
\hline Sum of skinfolds (mm) & $-5.7(-12.4$ to 1.6$)$ & 0 & $4.6(1.0$ to 8.4$)$ & $2.3(-2.3$ to 7.1$)$ & $4.5(0.2$ to 8.8$)$ & 0.01 \\
\hline Fat mass index $\left(\mathrm{kg} / \mathrm{m}^{5}\right)$ & $-5.9(-11.2$ to -0.2$)$ & 0 & $2.8(0.0$ to 5.7$)$ & $1.6(-2.0$ to 5.3$)$ & $3.3(0.0$ to 6.7$)$ & 0.01 \\
\hline $\mathrm{HbA}_{1 \mathrm{c}}(\%)$ & $0.4(-0.6$ to 1.3$)$ & 0 & $0.3(-0.2$ to 0.7$)$ & $0.6(0.0$ to 1.1$)$ & $0.0(-0.5$ to 0.5$)$ & 0.68 \\
\hline Glucose (mmol/L) & $-0.4(-1.6$ to 0.8$)$ & 0 & $0.5(-0.1$ to 1.0$)$ & $0.1(-0.6$ to 0.9$)$ & $0.2(-0.5$ to 0.8$)$ & 0.40 \\
\hline Insulin (mU/L) & $3.1(-6.2$ to 13.3$)$ & 0 & $9.0(4.2$ to 14.0$)$ & $4.9(-1.0$ to 11.2$)$ & 10.7 (5.1 to 16.7$)$ & $<0.001$ \\
\hline HOMA-IR & $3.2(-6.1$ to 13.3$)$ & 0 & $8.9(4.2$ to 13.9$)$ & $5.3(-0.6$ to 11.6$)$ & 10.5 (4.9 to 16.4$)$ & $<0.001$ \\
\hline Leptin (ng/mL) & $-9.0(-20.9$ to 4.6$)$ & 0 & 10.9 (3.8 to 18.5$)$ & $9.3(0.3$ to 19.1$)$ & $9.2(1.1$ to 18.0$)$ & 0.002 \\
\hline $\mathrm{C}$ reactive protein (mg/L) & $-23.5(-37.4$ to -6.6$)$ & 0 & $1.8(-7.4$ to 12.0$)$ & $0.8(-10.9$ to 14.1$)$ & $0.9(-9.7$ to 12.8$)$ & 0.26 \\
\hline Triglycerides (mmol/L) & $0.7(-5.0$ to 6.7$)$ & 0 & 3.2 (0.4 to 6.1$)$ & $2.2(-1.4$ to 5.9$)$ & $3.3(0.0$ to 6.7$)$ & 0.05 \\
\hline HDL cholesterol (mmol/L) & $-0.7(-3.7$ to 2.4$)$ & 0 & $-1.1(-2.5$ to 0.4$)$ & $0.1(-1.8$ to 2.0$)$ & $-1.6(-3.3$ to 0.1$)$ & 0.17 \\
\hline LDL cholesterol (mmol/L) & $-2.2(-5.8$ to 1.6$)$ & 0 & $-1.1(-2.9$ to 0.7$)$ & $-2.5(-4.7$ to -0.1$)$ & $-2.0(-4.1$ to 0.1$)$ & 0.06 \\
\hline Total cholesterol (mmol/L) & $-1.3(-3.8$ to 1.3$)$ & 0 & $-0.6(-1.8$ to 0.6$)$ & $-1.1(-2.6$ to 0.5$)$ & $-1.4(-2.8$ to 0.1$)$ & 0.10 \\
\hline \multicolumn{7}{|c|}{ Difference compared with 'An hour or less self-reported screen time' as the reference group $(95 \% \mathrm{Cl}), \mathrm{p}$ (difference) } \\
\hline Systolic BP (mm Hg) & $0.3(-1.3$ to 1.9$)$ & 0 & $0.2(-0.6$ to 0.9$)$ & $-0.2(-1.2$ to 0.7$)$ & $-0.5(-1.4$ to 0.4$)$ & 0.24 \\
\hline Diastolic BP (mm Hg) & $0.0(-1.4$ to 1.4$)$ & 0 & $0.0(-0.7$ to 0.7$)$ & $-0.2(-1.0$ to 0.7$)$ & $0.0(-0.8$ to 0.8$)$ & 0.85 \\
\hline Daily counts* & $11286(-10753$ to 33325$)$ & 0 & $-4720(-15012$ to 5572$)$ & $-13490(-26724$ to -256$)$ & $-6637(-18815$ to 5541$)$ & 0.05 \\
\hline $\begin{array}{l}\text { Daily time spent in MVPA } \\
\text { (mins)* }\end{array}$ & $2.0(-2.3$ to 6.3$)$ & 0 & $-0.3(-2.3$ to 1.7$)$ & $-2.6(-5.2$ to -0.1$)$ & $-1.2(-3.6$ to 1.2$)$ & 0.06 \\
\hline \multicolumn{7}{|c|}{$\begin{array}{l}\text { Differences (\% differences) adjusted for sex, age (quartiles), ethnic group, month and a random effect to allow for clustering within schools. } \\
\text { Percentage differences are presented for log-transformed variables. } \\
{ }^{*} \text { Based on } 2031 \text { participants with data for daily counts and daily time spent in MVPA, the numbers for each screen time group were as follows: none= }=80 \text {, an hour or less=729, } \\
1-2 \text { hours }=593,2-3 \text { hours }=270 \text {, more than } 3 \text { hours=359. } \\
\text { BP, blood pressure; HDL, high-density lipoprotein; HOMA, homeostasis model assessment; IR, insulin resistance; LDL, low-density lipoprotein; MVPA, moderate to vigorous physical } \\
\text { activity. }\end{array}$} \\
\hline
\end{tabular}


activity levels. The association with IR was substantially independent of adiposity (fat mass index).

Strengths of the present study include the large sample size, measurement of key T2D risk markers including $\mathrm{IR}^{1718}$ and the assessment of potential confounders including socioeconomic markers, pubertal status and physical activity. The children in the study were asked about the amount of time spent watching television, video or playing computer games which was appropriate to capture 'screen-time' when the study was conducted in 2004-2007. Studies in current settings would also need to take account of the use of more recently introduced electronic devices, for example, electronic tablets and smartphones, also potentially related to sedentary behaviour, which are now more widely used by children. Pubertal status was only assessed in girls in the present study, as evidence suggests that age of pubertal onset in boys is substantially higher than that in our study population. ${ }^{19-21}$ The CHASE study was powered to detect small $(\sim 0.2 \mathrm{SD})$ ethnic differences in risk markers presented here; however, we have been able to estimate associations between screen time and adiposity and cardiometabolic risk markers with narrow CIs, suggesting a high level of precision. Although the study response rate was modest, the characteristics of participants who provided a fasting blood sample were mostly similar to participants who did not. ${ }^{10}$ The participants in this study were recruited from three UK cities (London, Birmingham and Leicester) which together account for two-thirds of South Asians and black African-Caribbeans in the UK. The study sample is therefore likely to be representative of these ethnic minority populations; it may be less representative of white Europeans, as they were recruited from the same schools as the ethnic minority participants. However, the representativeness is unlikely to have biased the association between screen time and IR. ${ }^{22}$

While the present findings are of considerable potential public health interest, evidence from randomised controlled trials is needed to establish causality. Intervention studies in children showing decreased body size associated with reduced screen time are supportive of a causal effect, ${ }^{23}{ }^{24}$ although causal associations between screen viewing and early T2D risk factors remain to be established. Future studies could illuminate the causal pathways by which screen time manifests itself such as diet and lack of breaks in sedentary behaviour as well as decreased physical activity. Recommendations from the American Academy of Pediatrics (AAP) previously suggested that children should limit daily screen-time to $<2$ hours, ${ }^{25}$ although the most recent AAP guidance did not propose a time-limit, instead suggesting that parents should place consistent limits on hours per day of media use, ${ }^{26}$ proposing a more pragmatic interpretation given the pervasive use of electronic devices. ${ }^{7}$ However, such limitations, which may be beneficial for other aspects of health, must not overlook the underlying sedentary nature of screen-related activities and their potential impact on metabolic health. Our findings suggest that reducing screen time may be beneficial in reducing T2D risk factors, in both boys and girls and in different ethnic groups from an early age. This is particularly relevant, given rising levels of $\mathrm{T} 2 \mathrm{D}$, the early emergence of $\mathrm{T} 2 \mathrm{D}$ risk $^{1}$ and recent trends suggesting that screen-time-related activities are increasing in childhood ${ }^{7}$ and may pattern screen-related behaviours in later life. ${ }^{27}$

Acknowledgements The authors are grateful to the members of the CHASE study team and to all participating schools, pupils and parents.

Contributors All authors contributed substantially to the conception and design of this paper. PHW conceived, raised funding for and directed the CHASE study with help from DGC. All authors contributed to the collection of data used in this paper. CMN and ARR carried out the statistical analyses. CMN drafted the paper, which was critically appraised by all authors for intellectual content. All authors approved the final version to be published and agree to be accountable for all aspects of the manuscript.

Funding Diabetes prevention research at St George's, University of London is supported by the National Institute for Health Research Collaboration for Leadership in Applied Health Research and Care (CLAHRC) South London

(CLAHRC-2013-10022). Data collection was supported by grants from the Wellcome Trust (068362/Z/02/Z), the British Heart Foundation (PG/06/003) and by the National Prevention Research Initiative (NPRI). The Funding Partners for this NPRI award were: British Heart Foundation; Cancer Research UK; Department of Health; Diabetes UK; Economic and Social Research Council; Medical Research Council; Research and Development Office for the Northern Ireland Health and Social Services; Chief Scientist Office, Scottish Executive Health Department and Welsh Assembly Government.

\section{Competing interests None declared.}

Patient consent Parental/guardian consent obtained.

Ethics approval Multicentre Research Ethics Committee, Wales, UK.

Provenance and peer review Not commissioned; externally peer reviewed.

Data sharing statement Enquiries about use of the study dataset can be made by contacting Professor Peter Whincup, St George's, University of London, UK.

Open Access This is an Open Access article distributed in accordance with the terms of the Creative Commons Attribution (CC BY 4.0) license, which permits others to distribute, remix, adapt and build upon this work, for commercial use, provided the original work is properly cited. See: http://creativecommons.org/licenses/ by/4.0/

\section{REFERENCES}

1 International Diabetes Federation. IDF diabetes Atlas. 6th edn. Brussels, Belgium: International Diabetes Federation, 2013. https://www.idf.org/sites/default/files/EN_ 6E_Atlas_Full_0.pdf (accessed Mar 2016).

2 Davies SC, Barlow J. Our children deserve better: prevention pays. Annual Report of the Chief Medical Officer 2012. 2013.

3 Thorp AA, Owen N, Neuhaus $M$, et al. Sedentary behaviors and subsequent health outcomes in adults a systematic review of longitudinal studies, 1996-2011. Am J Prev Med 2011:41:207-15.

4 Grøntved A, Hu FB. Television viewing and risk of type 2 diabetes, cardiovascular disease, and all-cause mortality: a meta-analysis. JAMA 2011;305:2448-55.

5 Falbe J, Rosner B, Willett WC, et al. Adiposity and different types of screen time. Pediatrics 2013;132:e1497-1505.

6 Tremblay MS, LeBlanc AG, Kho ME, et al. Systematic review of sedentary behaviour and health indicators in school-aged children and youth. Int I Behav Nutr Phys Act 2011:8:98.

7 Rideout VJ, Foehr UG, Roberts DF. Generation $M^{2}$ media in the lives of 8 to 18-year-olds: a Kaiser family foundation study. Menlo Park, California: Henry J. Kaiser Family Foundation, 2010. https://kaiserfamilyfoundation.files.wordpress. com/2013/04/8010.pdf (accessed Jun 2016).

8 Donin AS, Nightingale CM, Owen CG, et al. Ethnic differences in blood lipids and dietary intake between UK children of black African, black Caribbean, South Asian, and white European origin: the Child Heart and Health Study in England (CHASE). Am J Clin Nutr 2010:92:776-83.

9 Owen CG, Nightingale CM, Rudnicka AR, et al. Ethnic and gender differences in physical activity levels among 9-10-year-old children of white European, South Asian and African-Caribbean origin: the Child Heart Health Study in England (CHASE Study). Int J Epidemiol 2009;38:1082-93.

10 Whincup PH, Nightingale CM, Owen CG, et al. Early emergence of ethnic differences in type 2 diabetes precursors in the UK: the Child Heart and Health Study in England (CHASE Study). PLoS Med 2010;7:e1000263.

11 Thomas C, Nightingale CM, Donin AS, et al. Socio-economic position and type 2 diabetes risk factors: patterns in UK children of South Asian, black African-Caribbean and white European origin. PLOS ONE 2012;7:e32619.

12 Nightingale CM, Rudnicka AR, Owen CG, et al. Are ethnic and gender specific equations needed to derive fat free mass from bioelectrical impedance in children of South Asian, black African-Caribbean and white European origin? Results of the assessment of body composition in children study. PLOS ONE 2013;8:e76426.

13 Andersen L, Dinesen B, Jorgensen PN, et al. Enzyme immunoassay for intact human insulin in serum or plasma. Clin Chem 1993;39:578-82.

14 Levy JC, Matthews DR, Hermans MP. Correct homeostasis model assessment (HOMA) evaluation uses the computer program. Diabetes Care 1998:21:2191-2.

15 Thomas C, Nightingale CM, Donin AS, et al. Ethnic and socioeconomic influences on childhood blood pressure: the Child Heart and Health Study in England. J Hypertens 2012;30:2090-7.

16 Taylor SJ, Whincup PH, Hindmarsh PC, et al. Performance of a new pubertal self-assessment questionnaire: a preliminary study. Paediatr Perinat Epidemiol 2001;15:88-94. 
17 Morrison JA, Glueck CJ, Horn PS, et al. Pre-teen insulin resistance predicts weight gain, impaired fasting glucose, and type 2 diabetes at age 18-19 y: a 10-y prospective study of black and white girls. Am J Clin Nutr 2008:88:778-88.

18 Hanley AJ, Williams K, Gonzalez C, et al. Prediction of type 2 diabetes using simple measures of insulin resistance: combined results from the San Antonio Heart Study, the Mexico City Diabetes Study, and the Insulin Resistance Atherosclerosis Study. Diabetes 2003;52:463-9.

19 Aksglaede L, Olsen LW, Sorensen TI, et al. Forty years trends in timing of pubertal growth spurt in 157,000 Danish school children. PLOS ONE 2008;3: e2728.

20 Euling SY, Herman-Giddens ME, Lee PA, et al. Examination of US puberty-timing data from 1940 to 1994 for secular trends: panel findings. Pediatrics 2008;121 (Suppl 3):S172-91.

21 Sørensen K, Aksglaede L, Petersen JH, et al. Recent changes in pubertal timing in healthy Danish boys: associations with body mass index. J Clin Endocrinol Metab 2010;95:263-70.
22 Pizzi C, De Stavola B, Merletti F, et al. Sample selection and validity of exposure-disease association estimates in cohort studies. J Epidemiol Community Health 2011;65:407-11.

23 Epstein $\mathrm{LH}$, Roemmich JN, Robinson JL, et al. A randomized trial of the effects of reducing television viewing and computer use on body mass index in young children. Arch Pediatr Adolesc Med 2008;162:239-45.

24 Wu L, Sun S, He Y, et al. The effect of interventions targeting screen time reduction: a systematic review and meta-analysis. Medicine (Baltimore) 2016;95:e4029.

25 Strasburger VC, Hogan MJ. Children, Adolescents, and the Media. American Academy of Pediatrics, 2013. http://pediatrics.aappublications.org/content/pediatrics/ 132/5/958.full.pdf (accessed Jun 2016).

26 Moreno MA, Chassiakos YR, Cross C. Media Use in School-Aged Children and Adolescents: Policy Statement. American Academy of Pediatrics; 2016. http://pediatrics.aappublications.org/content/138/5/e20162592 (accessed Jan 2017).

27 Biddle SJ, Pearson N, Ross GM, et al. Tracking of sedentary behaviours of young people: a systematic review. Prev Med 2010;51:345-51.

\section{ARCHIVIST \\ Breastfeeding and obesity}

A number of observational cohort studies, including some from the UK, have shown lower rates of long-term obesity in children who are breastfed for longer. This is one of the many potential benefits used to encourage hesitant new mothers. However all these studies are inevitably biassed by confounding: whatever it is that makes a mother choose to breastfeed is associated with other, sometimes unmeasurable, favourable parenting practices. Thus only a randomised trial could show any true benefit.

This has now effectively been done: a long-term study in Belarus recruited over 17000 mother-infant pairs who were already breastfeeding back in 1996-7 (Martin R et al. JAMA Pediatr 2017. doi:10.1001/jamapediatrics.2017.0698). Using a cluster-randomisation method, the 31 polyclinics that they were attending were randomised to undertake either a breastfeeding promotion scheme based on the internationally recognised Baby-Friendly Hospital initiative, or usual care as controls. The promotion scheme was very effective: $45 \%$ of those in it were exclusively breastfeeding at 3 months compared with $6 \%$ among the controls.

These children have now been followed to age 16 years: an age at which their adult obesity trajectory will probably have become inevitable. The results give no comfort to promoters of breastfeeding: there was no reduction in BMI or obesity rates in the intervention group, either in the earlier years or at 16. In fact if anything they were heavier than controls (OR for BMI $\geq 85^{\text {th }}$ centile $1.14 ; 95 \%$ CI 1.0 to 1.3 ). The only measurable benefit seen in the intervention group was more rapid vertical growth post-infancy. Blood pressure was also measured and did not differ significantly.

This is obviously disappointing but some caveats are needed: first, this was not a comparison of breast vs formula feeding from birth, as all received some breast milk, although for only a few weeks in the controls. Second, the social and cultural circumstances in Belarus may have been quite different to Western European and North American communities, where obesity is more prevalent anyway, and the results are not necessarily generalisable.

So breastfeeding, a practice so beneficial that it seems to prevent almost everything bad, has come up against obesity, a societal problem so intractable that almost no public health intervention seems to prevent it, and failed to deliver. We have to keep looking for other solutions.

Competing interests None declared.

Provenance and peer review Commissioned; internally peer reviewed.

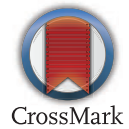

Accepted 9 May 2017

Published Online First 6 June 2017

Arch Dis Child 2017;102:616. doi:10.1136/archdischild-2017-313361. 